\title{
Septal mucoperiosteal flap for the repair of unilateral choanal atresia*
}

\author{
Ranko Mladina ${ }^{1}$, Ratko Prstašić ${ }^{1}$, Katarina Vuković ${ }^{1}$, Neven Skitarelić ${ }^{2}$ \\ Clinic for Otorhinolaryngology and Head and Neck Surgery Rebro, Clinical Hospital Center Zagreb, Zagreb, \\ Croatia \\ 2 Department of Otolaryngology Head and Neck Surgery, Zadar General Hospital, Zadar, Croatia
}

\begin{abstract}
SUMMARY
The authors present a new modality of endoscopic repair for unilateral choanal atresia. A 14-year old girl complained of right-sided nasal obstruction. Endoscopy and CT scans showed unilateral bony choanal atresia. The patient underwent endoscopic endonasal surgery. A mucoperiosteal flap at the posterior part of the septum in the left, healthy nasal cavity was fashioned and the denuded part of the septum was removed. The atretic choanae was resected and a unified posterior nasal opening was formed. The flap was spread over the posterior septal edge and adjusted to the opposite septal side.

One-year follow-up of the patient showed no stenosis of the choanae.
\end{abstract}

Key words: unilateral choanal atresia, endoscopic surgery, mucoperiosteal septal flap

\section{INTRODUCTION}

Various surgical approaches to choanal atresia have been suggested, including the transpalatal ${ }^{(1,2)}$, transeptal ${ }^{(3,4)}$ and endoscopic transnasal approach ${ }^{(5,6)}$. Nowadays, the endoscopic approach combining transnasal and posterior transeptal technique is considered as the gold standard. The most important part of the transeptal approach is the resection of the posterior third of the septum, i.e. the vomer, which seems to be the crucial step in preservation of patency of newly formed choana. This technique, first proposed by Eiken in 1911 according to Rudert ${ }^{(7)}$, enables a creation of one unified posterior nasal space. However, the problem of postoperative scaring arises, since large spots of bony structures remain denuded, uncovered by mucosa. In order to prevent uncontrolled scaring processes and consecutive stenosis, a great number of techniques for fashioning mucosal flaps that could permanently cover the raw bony areas have been described ${ }^{(5,7,8)}$.

We present a case of unilateral bony choanal atresia in which we have used our simple surgical modality for fashioning a mucosal flap that perhaps may help in decreasing the possibility of postoperative stenosis.

\section{CASE REPORT}

A 14-year old girl complained of complete right-sided nasal obstruction and permanent mucous secretion dating back from earliest childhood. Anterior rhinoscopy showed a collection of mucus in the right nasal cavity and a mucosal hypertrophy of the right inferior turbinate. Left nasal cavity looked normal.
The endoscopic examination of the right nasal cavity showed a complete absence of the choana (Figure 1).

Axial CT scans showed typical appearance of the unilateral bony choanal atresia. Radiological aspects of the surrounding anatomy (pterygoid plate etc.) showed no particular irregularities. The only difference between atretic and healthy side of the nose was the thickness of the bony structures in general at the atretic side.

Surgery was preformed under general anesthesia. Additional decongestion of nasal mucosa was obtained by means of cotton pledgets, soaked in a mixture of $5 \%$ solution of cocaine

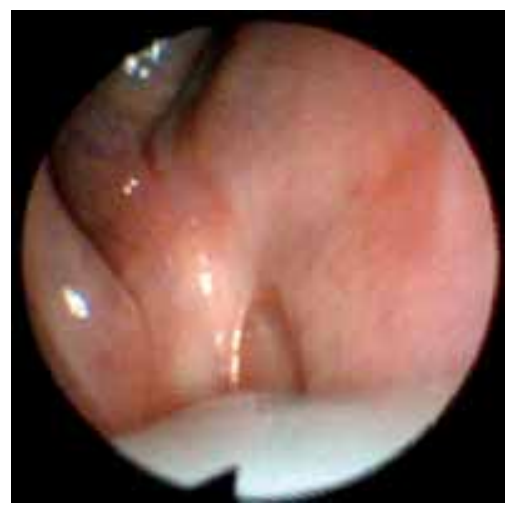

Figure 1. Endoscopic view of the right nasal cavity showing a complete absence of the choana. The black arrow at seven o'clock shows a typical collection of mucus at the bottom of the nasal cavity. 


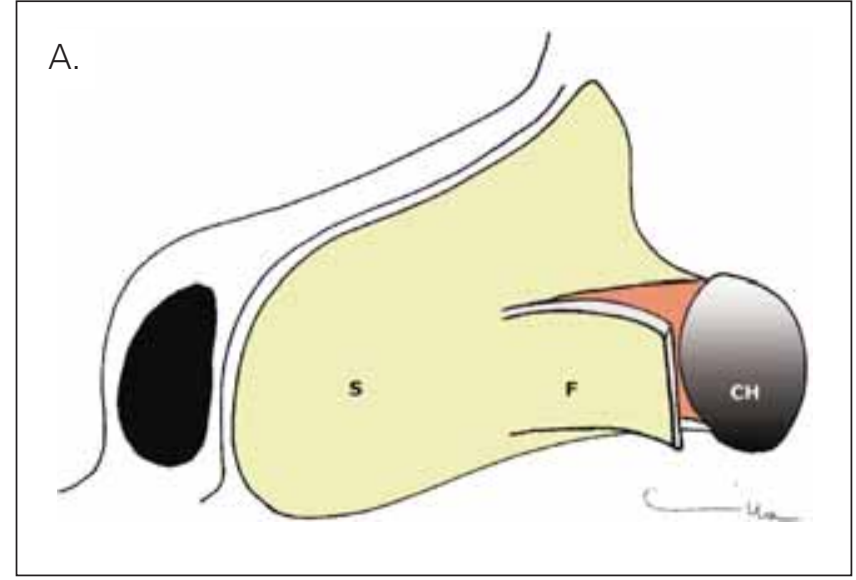

Figure 2a. The mucoperiosteal flap (F) has been elevated from the left side of the nasal septum (S), beginning from its choanal margin. $\mathrm{CH}$ choana.

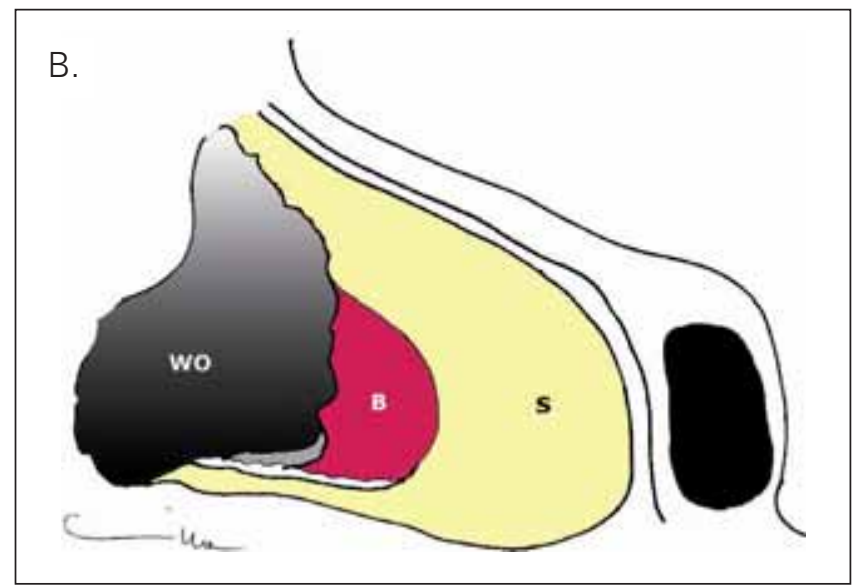

Figure 2b. Right nasal cavity. WO - wide opening after the removal of atretic tissue, B - bed for the mucoperiosteal flap coming from the other septal side, S - septum. The gray-coloured region - inferior part of the left, normal choanae.

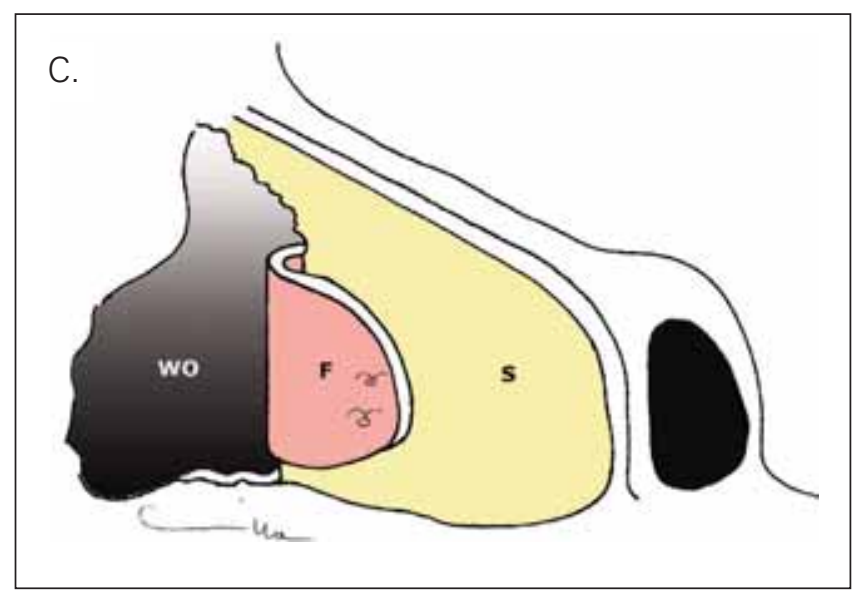

Figure 2c. Right nasal cavity. WO - wide opening, F - mucoperiosteal flap coming from the other septal side, S - septum. The flap has been fixed by means of two 3-0 stitches. and epinephrine (5:1) and an endoscopically guided infiltration of local anesthesia of the posterior parts of the nasal septum (bilaterally) and atretic choana. The endoscopic endonasal surgical technique was performed, using a $4 \mathrm{~mm} 30^{\circ}$ endoscope. A mucoperiosteal flap at the posterior part of the septum in the left, healthy nasal cavity was elevated by means of two horizontal incisions connected by the vertical incision along the posterior septal edge (Figure 2a). The posterior part of the septum was then fractured with a curette, and its mucosal and bony remnants were completely removed. A small area of denuded remaining septal skeleton at the right side was created as a future bed for the flap. The atretic choana was then opened by means of a curette and the diamond burr and connected to the resected area of the posterior part of septum, thus forming a unified, wide posterior nasal opening (Figure $2 b)$. The mucoperiosteal flap was than used as a biological bandage of the rough posterior septal edge and adjusted to the previously prepared bed at the opposite septal side. The flap was fixed with two stitches (Figure 2c). Instead of nasal packing, the flap was gently covered and protected by means of thin silicone septal sheets which were left in place for two weeks in order to allow an optimal and safe adherence of the flap to the bony skeleton of the nasal septum. The sheets were meticulously sutured at the septum by means of two 4-0 PDS stitches. The patient is doing well more than one year after surgery (Figure 3).

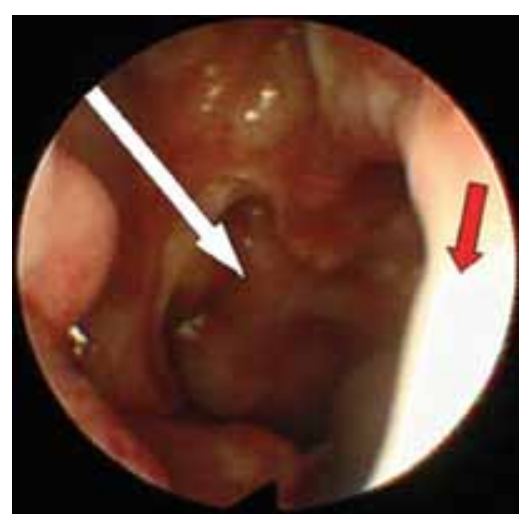

Figure 3: Endoscopic view of the posterior part of the right nasal cavity one year after the surgery. Red arrow-remnant of the nasal septum completely epithelized with no granulations. White arrow indicates a large, unified, wide posterior nasal opening.

\section{DISCUSSION}

The treatment of choanal atresia should result in enduring functional nasal breathing with minimal perioperative and postoperative complications. The endonasal endoscopic technique combining transnasal with posterior transeptal technique has become a gold standard in surgery for choanal atresia. It provides direct visualization of the surgical field without need for an open approach, offering advantages of surgical precision in bone resections of the posterior part of septum and the atretic area. This surgical technique minimizes the trauma of 
the surrounding tissues thus decreasing the rate of postoperative stenosis and the need for revision surgery ${ }^{(5,7,9)}$. Samadi et al ${ }^{(10)}$ found out in a retrospective comprehensive study involving a period of time between year 1979 -1998 that unilateral choanal atresia required, on average, 2.7 total procedures, including dilatation and removal of the stents! They also found out that nearly all children underwent correction by means of an endonasal endoscopic surgery. In addition, Rombaux et al. (11) reported mean surgical repair per patient before obtaining patent choana to be 1.14 for those operated by means of endoscopic endonasal surgery technique, whereas "historic" cases (operated between year 1990-1998) which were operated without endoscopic control required 1.89 procedures. The patency of newly created choana at twelve months was much higher in endoscopic group than in non-endoscopic one $(85.7 \%$ and $47.3 \%$, respectively). The formation of granulation tissue is a part of the natural healing process of the denuded bone.

On the other hand, stenosis can easily occur as a result of an excessive growth of granulation tissue. Various authors proposed different surgical techniques using mucosal remnants to form flaps in order to cover with normal epithelium as much of the raw surfaces as possible. Stankiewicz ${ }^{(5)}$ used the mucosa of the pharyngeal side and the nasal side of the atresia to cover the raw areas of the bony resections and recommended the use of postoperative nasal packing and stents. Rudert ${ }^{(7)}$ described the technique of preservation of mucosa of the pharyngeal part of the atresia and fashioning of the protective flaps in order to cover raw bony areas, followed by Merocel nasal packing postoperatively. Stamm and Pignatari ${ }^{(8)}$ used the septal mucosa and mucosa of the atretic nasal faces and folded them over to cover the raw areas exposed from the removal of the posterior septum and atretic plates. They also insisted on the use of nasal packing in order to keep the flaps in place. Contrary to this, many authors describe techniques that exclude the use of stents and nasal packing ${ }^{(9,12)}$, considering the possible disadvantages of their long-term use: the irregularity of mucociliary drainage, bacterial infections and potentially increased scar tissue formation, contributing to stenosis and leading to revision surgery. Gosepath et al. ${ }^{(13)}$ showed that the incidence of revisions because of postoperative stenosis was three times less in patients who did not have any stents. The decision whether to use stents or not remains a subject of controversy in surgical management of choanal atresia. We abandoned the use of stents in choanal atresia surgery many years ago since we noticed increased tendency of scar and granulation tissue formation, remarkably greater than in patients without stents. In the surgical modality described in this paper we decided to cover the flap for a while just to protect it from the direct airstream, from drying (mummification even possible), infection, and probable disturbances in the process of local tissues healing because of physical reasons like uncontrolled nose blowing, sneezing etc.

Some authors emphasized the importance of the lateral borders of the resection area, considering those parts to be the ori- gin of the main granulation tissue formation ${ }^{(14)}$. Contrary to that, the idea for our modality was based on our clinical observation that the main scar tissue formation, which usually ruins the initial success of the operation, happens in fact at the newly created posterior septal margine. This is why we insisted on covering the well-defined area with specially created mucoperiosteal septal flap.

Our technique was quick, safe, without any perioperative or postoperative complications and resulted in fast postoperative recovery. This technique seems to be very effective and elegant but requires skilled endoscopic rhino-surgeon. So far it showed no need for revision surgery and adequate functional breathing was maintained during the entire follow-up period.

\section{REFERENCES}

1. Owens H. Observations in treating twenty-five cases of choanal atresia by the transpalatine approach. Laryngoscope 1965; 75: 84104.

2. Pirsig W. Surgery of choanal atresia in infants and children: historical notes and updated review. Int J Pediatr Otorhinolaryngol 1986; 11: 153-170.

3. Carpenter RJ, Neel HB. Correction of congenital choanal atresia in children and adults. Laryngoscope 1987; 87: 1304-1311.

4. Krespi YP, Husain S, Levine TM, Reede DL. Sublabial transseptal repair of choanal atresia or stenosis. Laryngoscope 1987; 97: 14021406.

5. Stankiewicz JA. The endoscopic repair of choanal atresia. Otolaryngol Head Neck Surg. 1990; 103: 931-937.

6. Deutsch E, Kaufman M, Eilon A. Transnasal endoscopic management of choanal atresia. Int Pediatr Otorhinolaryngol 1997; 40: 1926.

7. Rudert H. Kombiniert transseptale-transnasale chirurgie einseitiger choanalatresien ohne verwendung von platzhaltern. Laryngo Rhino Otol 1999; 78: 697-702.

8. Stamm AC, Pignatari SSN. Nasal septal cross-over flap technique: a choanal atresia micro-endoscopic surgical repair. Am J Rhinol 2001; 15: 143-148.

9. Cedin AC, Rocha Jr FP, Depperman MB, Moraes Manzano PA, Murao M, Shimuta AS. Transnasal endoscopic surgery of choanal atresia without the use of stents. Laryngoscope 2002; 112: 750-752.

10. Samadi DS, Shah UK, Handler SD. Choanal atresia: a twenty-year review of medical comorbidities and surgical outcomes. Laryngoscope 2003; 113: 254-258.

11. Rombaux P, de Toeuf C, Hamoir M, Eloy P, Bertrand B, Veykemans F. Transnasal repair of unilateral choanal atresia. Rhinology 2003; 41: 31-36

12. Abbeele TVD, Francois M, Narcy P. Transnasal endoscopic treatment of choanal atresia without prolonged stenting. Arch Otolaryngol Head Neck Surg 2002; 128: 936-40.

13. Gosepath J, Santamaria VE, Lippert BM, Mann WJ. Forty-one cases of congenital choanal atresia over 26 years-retrospective analysis of outcome and technique. Rhinology 2007; 45: 158-163.

14. Holland BW, McGuirt WF. Surgical management of choanal atresia. Arch Otol Head Neck Surg 2001; 127: 1375-1380.

Neven Skitarelić, MD, PhD

Put Murvice 33

23000 Zadar

Croatia

E-mail: neven.skitarelic@zd.t-com.hr 